\title{
CONTINUOUS FORMS OF GAUSS-PÓLYA TYPE INEQUALITIES INVOLVING DERIVATIVES
}

\section{LUdMILA NikOlOVA AND SANJA VAROŠANEC}

Abstract. The main aim of this paper is to give a continuous form of the Gauss-Pólya type inequalities i.e. to give inequalities involving infinitely many functions. We consider inequalities which involve derivatives and which structure is related to the Hölder inequality. Also, some properties of the corresponding functionals are given.

Mathematics subject classification (2010): 26D10, 26D15.

Keywords and phrases: The Gauss-Pólya inequalities, the Hölder inequality, linear functional, monotone function.

\section{REFERENCES}

[1] S. Abramovich, J. PeČarić, S. Varošanec, New generalization of Gauss-Polya's inequality, Math. Inequal. Appl. 1, (1998), 331-342.

[2] H. Alzer, An extension of an inequality of G. Pólya, Bul. Inst. Politeh. Iasi Sect. I. Mat. Mec. Teor. Fiz. Tomul XXXVI (XL), Fasc. 1-4, (1990), 17-18.

[3] C. F. GaUss, Theoria combinationis observationum, German translation in Abhandlungen zur Methode der kleinsten Quadrate. Neudruck. Würzburg 1964, pp.9 and 12.

[4] E. G. Kwon, Extension of Hölder's inequality(I), Bull. Austral. Math. Soc. 51, (1995), 369-375.

[5] L. Nikolova, L.-E. Persson, On interpolation between $X^{p}$ spaces, Function spaces, Differential Operators and Nonlinear Analysis, Pitman Res. Notes in Math. 211, 1989.

[6] L. Nikolova, L.-E. Persson, S. Varošanec, Continuous forms of classical inequalities, Mediterr. J. Math. 13(5), (2016), 3483-3497.

[7] J. PeČARIĆ, S. VARošAneC, A generalization of Pólya's inequalities, Inequalities and Applications, World Scientific Publishing Company, Singapore, 1994, pp. 501-504.

[8] G. Pólya AND G. SzEGÖ, Aufgaben und Lehrsätze aus der Analysis, Vols. I and II. Berlin, Springer Verlag, 1925.

[9] S. VAROŠANEC, A remark on Volkov's result, Rad HAZU, [470]12, (1995), 199-203.

[10] S. VARošAneC, Superadditivity of functionals related to Gauss' type inequalites, Sarajevo J. Math. 10(22), No.1 (2014), 37-45.

[11] S. VARošAneC, Functionals related to Gauss-Pólya type inequalites involving derivatives, Results Math. (2019) 74:53. https://doi.org/10.1007/s00025-019-0983-1.

[12] S. VArošaneC, J. PeČArić, Gauss' and Related Inequalities, Z. Anal. Anwend. 14, (1995), 175183.

[13] V. N. Volkov, Utočnenie neravenstva Gel'dera, Mosk. Gos. Ped. Inst. im V.I. Lenina. Učen. Zap.Neravenstva, 460, (1972), 110-115. 